\title{
Detection and Removal of Non-responsive Channels and Trials in Evoked Potentials using Median test
}

\author{
V. Adinarayana Reddy ${ }^{1}$, P. Chandra Sekhar Reddy ${ }^{2}$, G. Hemalatha ${ }^{3}$, B. Anuradha ${ }^{4}$, \\ T. Jaya Chandra Prasad ${ }^{5}$ \\ ${ }^{1}$ RVP Engineering College for Women, Cuddapah \\ reddyadi2003@yahoo.co.in \\ ${ }^{2}$ JNTUH, Hyderabad \\ drpcsreddyegmail.com \\ ${ }^{3}$ KSRM College of Engineering, Cuddapah \\ latha.gumireddy@gmail.com \\ ${ }^{4}$ SVU College of Engineering, Tirupati \\ anubhumąyahoo.com \\ ${ }^{5}$ RGM College of Engineering \& Technology, Nandyal \\ jp_talarierediffmail.com
}

\begin{abstract}
The primary goal of this research work is to detect and remove non responsive channels and trials in evoked potentials by tracing out the signals with very low energy. This is done by calculating the energy of the average evoked potential of each channel, and the energy of the average evoked potential of each trial. Then channel wise and trial wise median test is conducted to detect and remove non-responsive channels and trials. An attempt has been made to apply these techniques to 14-channel visual evoked potentials (VEPs) obtained from four different subjects.
\end{abstract}

\section{KEYWORDS}

evoked potentials, energy, median..

\section{INTRODUCTION}

Evoked potentials (EPs) are usually considered as the time locked and synchronized activity of a group of neurons that add to the background electro-encephalogram (EEG). Evoked Potentials indicate how well the brain is processing stimuli from the sense organs (eg. eyes, ears or skin) and can help diagnose illnesses. An evoked potential (EP) is a signal that is generated as a result of the transmission of information induced by the application of a sensory stimulus to a sensory pathway. Examples of such stimuli are electric stimuli, visual stimuli, and auditory stimuli [1]. The application of a stimulus invokes a sequence of action potentials that is transmitted via a nervous pathway to the central nervous system (CNS).

The activation of different parts in the nervous pathway leads to variations in the electromagnetic field that can be recorded on the scalp. Using surface electrodes a sequence of positive and DOI : $10.5121 /$ sipij.2011.2404 
Signal \& Image Processing : An International Journal (SIPIJ) Vol.2, No.4, December 2011

negative peaks can be recorded; such a sequence is called a sensory evoked potential. These peaks are characterized by their amplitude and time after the stimulus, at which they occur the (post stimulus) latency. Evoked potentials are simultaneously recorded on the scalp with the spontaneous EEG. The EEG signal has much larger amplitude than the evoked potential. Averaging techniques are used to extract the signal related to the stimulus and reduce the amplitude of the ongoing EEG signal, which is treated as back ground noise while recording evoked potentials [2]-[4].

Evoked potentials are used extensively in the study of human brain functions and in clinical investigations to study normal and abnormal brain functions. They are used to test conduction in the visual, auditory, and somatosensory systems. During surgery they can be used to monitor the condition of structures at the operative site. Sensory evoked potentials can also be used for monitoring effects of anesthetics on the central nervous system (CNS). The choice of stimulus type to be used depends on the part of the nervous system to be investigated and the circumstances under which measurements are to be made.

Visual evoked potentials are very useful in detecting blindness in patients those cannot communicate, such as babies or animals. If repeated stimulation of the visual field causes no changes in EEG potentials then the subject's brain is probably not receiving any signals from his/her eyes. Other applications include the diagnosis of optic neuritis, which causes the signal to be delayed. Artifacts in EP waveform recordings typically result from voltage changes due to eye blinks, eye movements, muscle activities, and power line noise. Fig.1(a) shows visual evoked potential recording setup where pattern reversal method is used as stimulus, and Fig.1(b) shows a typical visual evoked potential.

A class of stimuli such as visual, auditory or somatosensory signal affects a particular group of neurons that can be detected by respective channel sensors placed on the scalp. Evoked potential response to each trial of the stimuli depends on the concentration of the subject on the applied stimuli at that particular time.

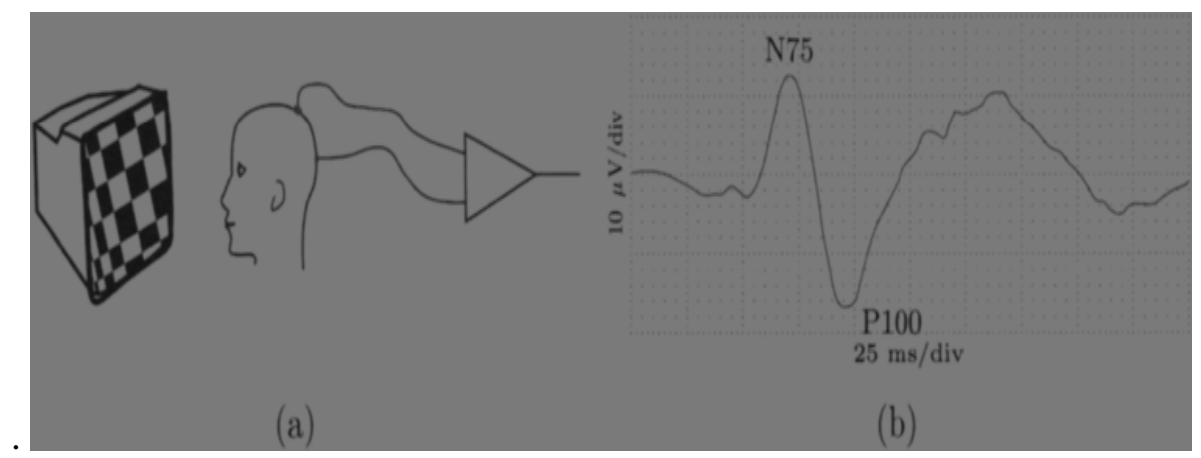

Fig.1. Visual evoked potentials. (a) Recording setup using pattern reversal method as stimulation. (b) Typical VEP morphology.

\section{RELATED WORK}

Several researches are going on to improve the quality of bio-medical signals. Errors in averaging of small signal samples can be reduced more efficiently by using median rather than mean [6]-[8]. Artifacts in visual evoked potentials caused by eye movement, eye blink, external noise, internal noise of recording instruments, etc., are removed by using different techniques such as blind 
component separation, multichannel median test, standard deviation and kurtosis tests, etc., [14][16].

\section{Median Test}

This work proposes median test to detect and remove non-responsive channels and trials in evoked potentials.

\subsection{Removal of Non Responsive Channels}

If a channel has stuck at fault, the EPs of that channel are discarded from further analysis. Some of the channels may not respond to a particular class of stimulus. In such cases, the non responsive channels may be detected as follows. $k^{\text {th }}$ sample of $\mathrm{N}-$ trial average evoked potential of each of the $\mathrm{M}$ channels is

$$
Z_{m / c}(k)=\frac{1}{N} \sum_{n=1}^{N} Z_{m / c ; n}(k), \begin{aligned}
& m=1,2, \ldots, M \\
& k=1,2, \ldots, K
\end{aligned}
$$

Where $Z_{m / c ; n}(k)$ is the $k^{\text {th }}$ sample of $n^{\text {th }}$ trial of $m^{\text {th }}$ channel evoked potential in response to stimulus $c$. Energy $E_{Z_{m}}$ of $\mathrm{N}$ - trial average of each channel $m$ is calculated as

$$
E_{Z_{m / c}}=\sum_{k=1}^{K} Z_{m / c}^{2}(k) \quad m=1,2, \ldots, M
$$

Let $\bar{E}_{Z_{c h l c}}$ be the median, $E_{Z_{c h / c} \max }$ be the maximum value and $E_{Z_{c h l c} \min }$ be the minimum value of $E_{Z_{1 / c}}, E_{Z_{2 / c}}, \ldots, E_{Z_{M / c}}$.

Define $d_{c h / c}=E_{Z_{c h / c} \max }-\bar{E}_{Z_{c h / c}}$ as the distance between the median and maximum value of energy of $\mathrm{M}$ - channels.

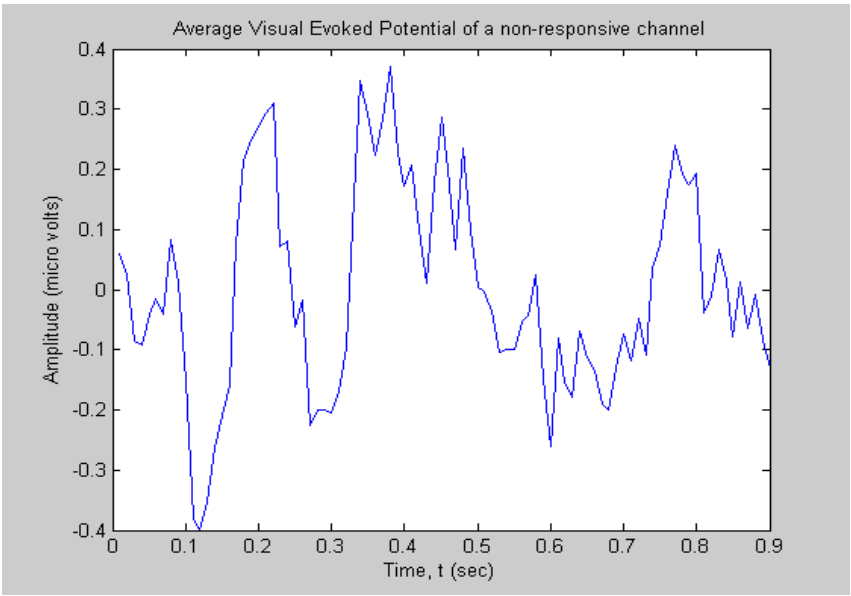

Fig. 2. Fig. Average VEP of a non-responsive channel. 
The channels providing average evoked potentials with energy less than $\bar{E}_{Z_{c h l c}}-d_{c h / c}$ are detected as non responsive channels, and removed from the channel averaging process. This will improve the peaks average EP responses. Fig.2 shows an example of average VEP of a non responsive channel, whereas Fig.3 shows that of a channel with good response to the applied stimulus $c$. Fig.4 shows a comparison of average VEPs including and excluding non responsive channels.

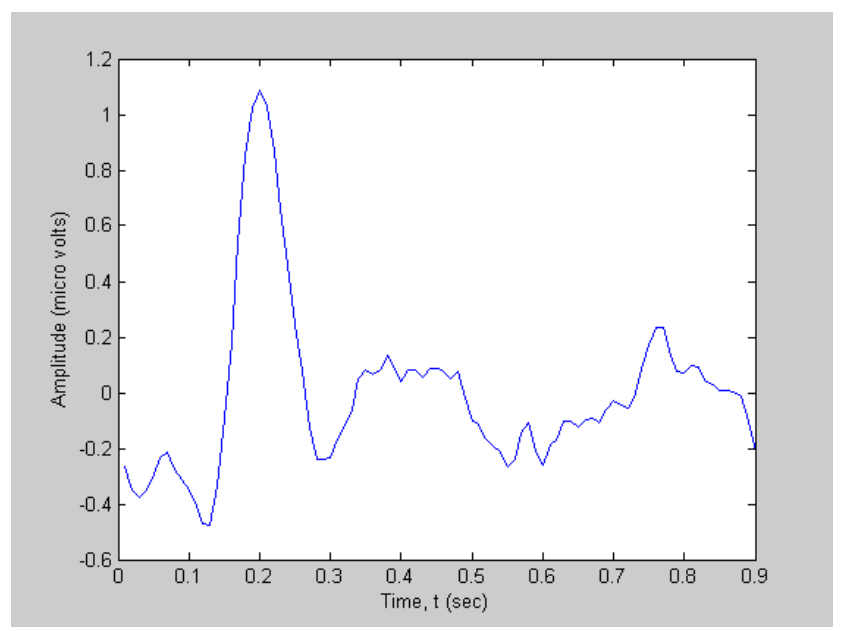

Fig. 3. Average VEP of a channel with good response

\subsection{Removal of Non Responsive Trials}

If the subject is not ready, or diverted from the stimulus, then evoked potentials of some trials may be non responsive. In such cases, the non responsive trials may be detected as follows. $k^{\text {th }}$ sample of $\mathrm{M}$ - channel average evoked potential of each of the $\mathrm{N}$ trials is

$$
Z_{c ; n}(k)=\frac{1}{M} \sum_{m=1}^{M} Z_{m / c ; n}(k), \begin{aligned}
& n=1,2, \ldots, N \\
& k=1,2, \ldots, K
\end{aligned}
$$

Where $Z_{m / c ; n}(k)$ is the $k^{\text {th }}$ sample of $n^{\text {th }}$ trial of $m^{\text {th }}$ channel evoked potential in response to stimulus $c$. Energy $E_{Z_{n}}$ of $\mathrm{M}-$ channel average of each trial $n$ is calculated as

$$
E_{Z_{c ; n}}=\sum_{k=1}^{K} Z_{c ; n}^{2}(k) \quad n=1,2, \ldots, N
$$

Let $\bar{E}_{Z_{t r / c}}$ be the median, $E_{Z_{t r / c} \max }$ be the maximum value and $E_{Z_{t r / c} \min }$ be the minimum value of $E_{Z_{c ; 1}}, E_{Z_{c ; 2}}, \ldots, E_{Z_{c ; N}}$.

Define $d_{t r / c}=E_{Z_{t r / c} \max }-\bar{E}_{Z_{t r / c}}$ as the distance between the median and maximum value of energy of $\mathrm{N}$ - trials. 
The trials providing average evoked potentials with energy less than $\bar{E}_{Z_{c ; t r}}-d_{t r / c}$ are detected as non responsive trials, and removed from the trial averaging process.

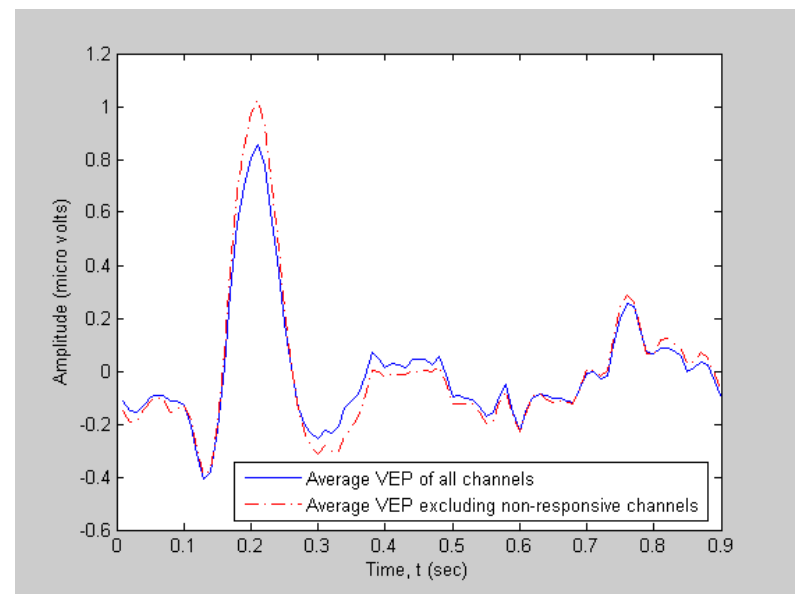

Fig.4. Comparison of VEPs with and without non responsive channels

Fig.5 shows an example of average EP of a non responsive trial. Removal of such trials will improve the peaks of average VEP responses, on addition to that provided by removing non responsive channels. Fig.6 shows average VEP of a non responsive trail after the removal of non responsive channels. Fig.7 shows a comparison of average VEPs including and excluding non responsive trials. A comparison of average VEPs with and without both non responsive channels and trials is shown in Fig.8.

Quality factor (Q-factor), $\theta=1-\hat{\theta}$

Where $\hat{\theta}=\frac{a}{N}$

$a=$ No. of non-responsive channels (trials)

$N=$ No. of tested channels (trilas) 
Signal \& Image Processing : An International Journal (SIPIJ) Vol.2, No.4, December 2011

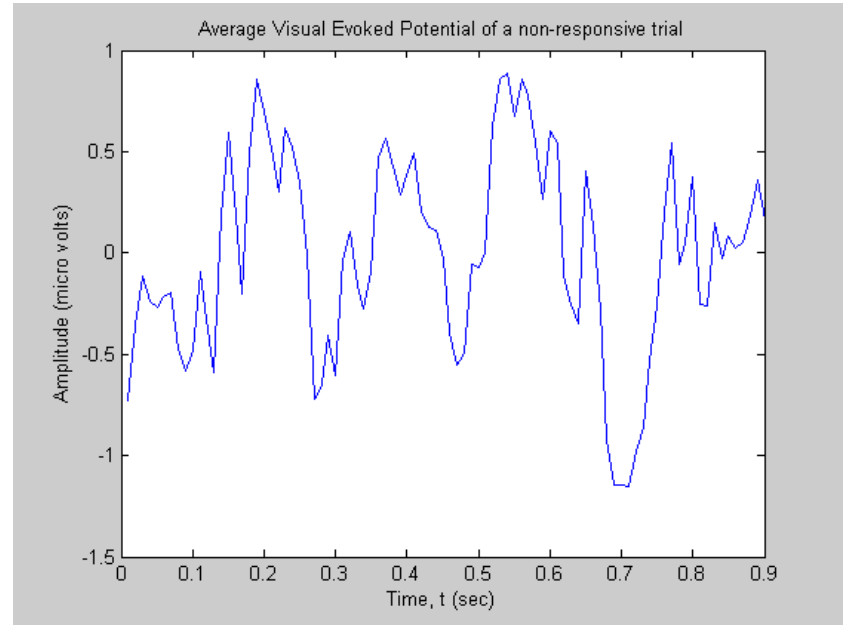

Fig.5. Average VEP of a non-responsive trial.

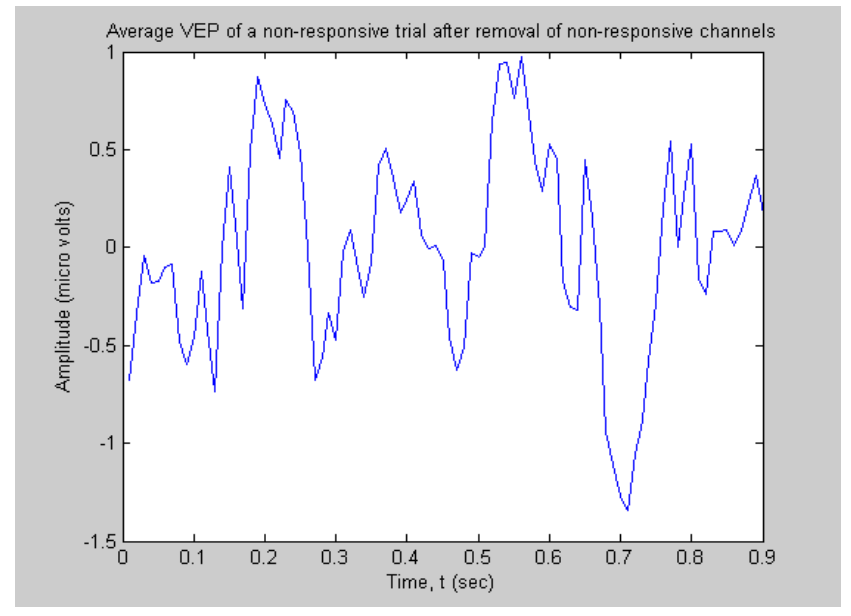

Fig.6. Average VEP of a non-responsive trial after removal of non responsive channels.

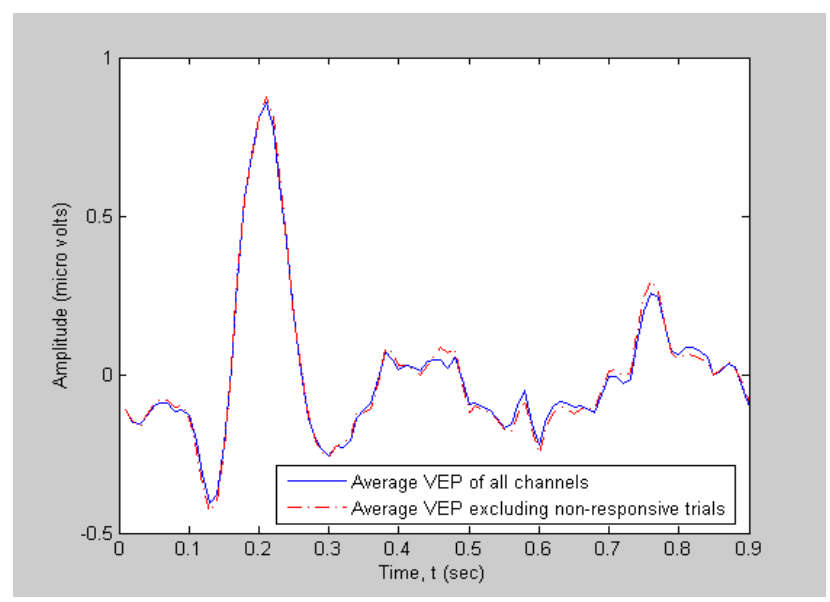

Fig.7. Comparison of VEPs with and without non responsive trials 


\section{SiMULATION AND RESULTS}

The median test was applied to 14-channel 71-trial VEP ensembles acquired from four different subjects. Channel wise and trial wise average EPs having low energies were detected and removed while classifying the EPs. The following table shows no. of non-responsive channels and trials and quality factors related to visual evoked potentials of a typical subject.

\begin{tabular}{|l|l|}
\hline No. of non-responsive channels detected & 2 \\
\hline No. of non-responsive trials detected & 4 \\
\hline No. of non-responsive trials detected after removal of non responsive channels & 3 \\
\hline Channel wise Q - factor before removal of non-responsive channels & $85.71 \%$ \\
\hline Trial wise Q - factor before removal of non-responsive trials & $94.37 \%$ \\
\hline Over all Q - factor before removal of non-responsive channels and trials & $80.89 \%$ \\
\hline $\begin{array}{l}\text { Trial wise Q - factor after removal of non-responsive channels but before removal } \\
\text { of non-responsive trials }\end{array}$ & $95.78 \%$ \\
\hline Over all Q - factor after removal of non-responsive channels and trials & $100 \%$ \\
\hline
\end{tabular}

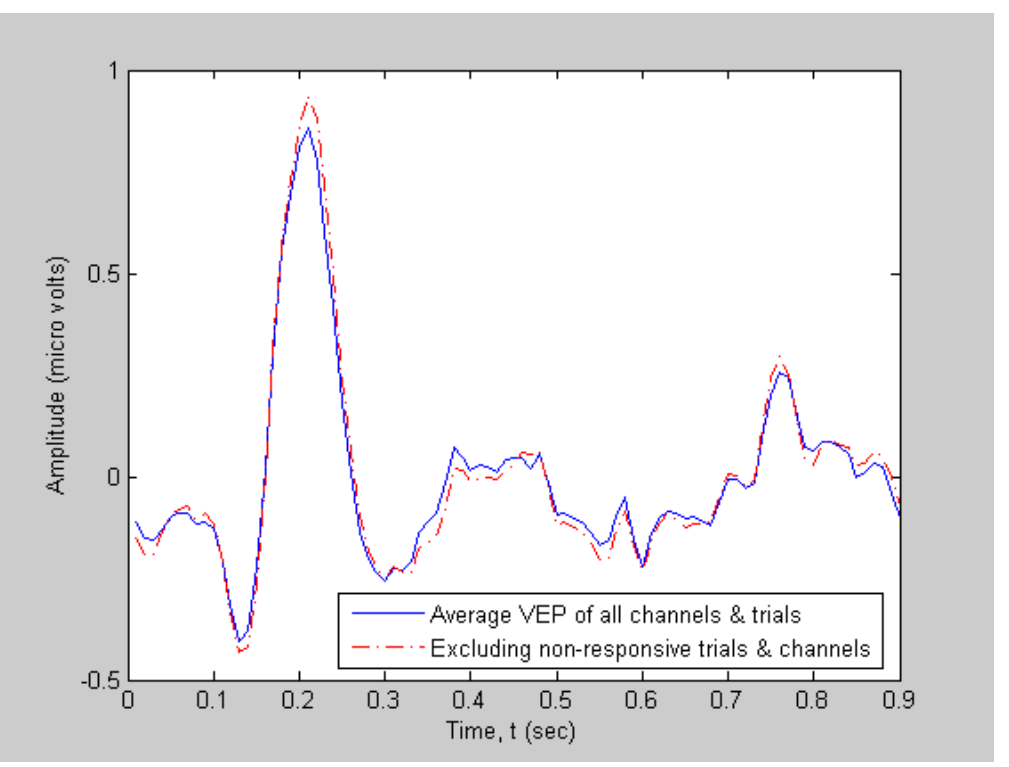

Fig.8. Comparison of VEPs with and without nonresponsive channels \& Trials.

\section{CONCLUSIONS}

The primary objective of this work is to identify and reject non responsive channels and trials in the acquisition of evoked potentials. The proposed method is applied to visual evoked potentials. Energy of average VEP of each channel, and of each trial is obtained. Then non responsive channels and trials are detected and removed by using channel wise and trial wise median test respectively. This improves the peaks of average VEPs and hence classifier performance. 
Signal \& Image Processing : An International Journal (SIPIJ) Vol.2, No.4, December 2011

\section{REFERENCES}

[1] Rodrigo Quan Quroga, Evoked potentials Encyclopedia of Medical Devices and Instrumentation, Second Edition, John Wiley \& Sons, Inc.(2006).

[2] Leif Sarnmo and Pablo Laguna, Bioelectrical Signal Processing in Cardiac and Neuralogical Applications, Elsevier Academic Press.

[3] Karl Friston, "A theory of cortical responses," Philosophical Transactions of the Royal Society, published online (29 April 2005) 815-836.

[4] T.D. Lagerlund, F.W. Sharbrough, N.E. Busacker, "Spatial filtering of multichannel electroencephalo-graphic recordings through principal component analysis by singular value decomposition,” J. of Clin. Neurophysiol. 14 (1997) 73-82.

[5] S. Casarotto, A.M. Bianchi, S. Cerutti, G.A. Chiarenza, "Principal component analysis for reduction of ocular artifacts in event-related potentials of normal and dyslexic children," Clin. Neurophysiol. 115 (3) (2004) 609-619.

[6] R. P. Borda and J. D. Frost, "Error reduction in small sample averaging through the use of the median rather than the mean," lectroencephal. Lin. Neurophysiol., vol. 25, pp. 391-392, 1968.

[7] G. D. Dawson, "A summation technique for detecting small signals in a large irregular background," J. Physiol. (London), vol. 115, p. 2, 1951.

[8] G. D. Dawson, "A summation technique for the detection of small evoked potentials," Electroencephal. Clin. Neurophysiol., vol. 6, pp. 65-84, 1954.

[9] H. Cecotti, "Classification of Steady-State Visual Evoked Potentials based on the Visual Stimuli Duty Cycle," IEEE, 3rd International Symposium on Applied Sciences in Biomedical and Communication Technologies (ISABEL), 2010.

[10] Zhiguo Zhang, Keith D. K. Luk, and Yong Hu, "Identification of detailed time-frequency components in somatosensory evoked potentials," IEEE Transactions on Neural Systems and Rehabilation Engineering, Vol. 18, NO. 3, pp.245-254, 2010.

[11] Ruben Gaitan-Ortiz, Oscar Yanez-Suarez, and Juan M Cornejo-Cruz, "Evoked potentials SNR maximization by PCA and genetic algorithms," Proceedings of the 5th International IEEE EMBS Conference on Neural Engineering Cancun, Mexico, April 27 - May 1, 2011, pp. 166-169, 2011.

[12] H. Nezamfar, U. Orhan1, D. Erdogmus, K.E. Hild, S. Purwar1, B. Oken and M.Fried-Oken, "On visual evoked potentials in EEG induced by multiple pseudorandom binary sequences for brain computer interface design," IEEE International Conference on Acoustics, Speech and Signal Processing (ICASSP), pp. 2044-2047, 2011.

[13] Gary Garcia-Molina, and Danhua Zhu, "Optimal spatial filtering for the steady state visual evoked potential: BCI application,'Proceedings of the 5th International IEEE EMBS Conference on Neural Engineering Cancun, Mexico, April 27 - May 1, 2011, pp. 156-160, 2011.

[14] C.A. Joyce, I.F. Gorodnitsky, M. Kutas, "Automatic removal of eye movement and blink artifacts from EEG data using blind component separation," Psychophysiology 41 (2) (2004) 313-325.

[15] Hyunseok Kook, Lalit Gupta, Srinivas Kota, Dennis Molfese, H. Lyytinen, “An offline/real-time artifact rejection strategy to improve the classification of multi-channel evoked potentials," Pattern Recognition (2008), pp. 1985-1996. 
Signal \& Image Processing : An International Journal (SIPIJ) Vol.2, No.4, December 2011

[16] V. Adinarayana Reddy, P. Chandra Sekhar Reddy, G. Hemalatha, T. Jayachandra Prasad, "Removal of Artifacts in Multi-channel Visual Evoked Potentials," International Journal of Modern Engineering Research (IJMER) www.ijmer.com Vol.1, Issue.2, pp-413-417 ISSN: 2249-6645 .

[17] L. Gupta, D.L. Molfese, R. Tammana, P.G. Simos, "Non-linear alignment and averaging for estimating the evoked potential," IEEE Tran. Biomed. Eng. 43 (4) (1996) 348-356.

[18] L. Gupta, zz J. Phegley, D.L. Molfese, "Parametric classification of multichannel evoked potentials," IEEE Trans. Biomed. Eng. 49 (8) (2002) 905-911 49(9) (2002) 1070.

[19] L. Gupta, B. Chung, M.D. Srinath, D.L. Molfese, H. Kook, "Multichannel fusion models for the parametric classification of differential brain activity," IEEE Trans. Biomed. Eng. 52 (11) (2005) 1869-1881.

[20] R.J. Croft, R.J. Barry, "Removal of ocular artifact from EEG: a review," Clin. Neurophysiol. 30 (1) (2000) 5-19.

[21] G.L. Wallstrom, R.E. Kass, A. Miller, J.F. Cohn, N.A. Fox, "Automatic correction of ocular artifacts in the EEG: a comparison of regression based and component-based methods," Int. J. Psychophysiol. 53 (2) (2004) 105-119.

[22] R. J. Chabot and E. R. Jhon, "Normative evoked potential data," in Handbook of Electroencephalography and Clinical Electrophysiology: Clinical Applications of Computer Analysis of EEG and other Neurophysiological Signals, ch. 1, pp. 263-309, Amsterdam/New York: Elsevier, 1986.

[23] T. W. Picton, O. G. Lins, and M. Scherg, "The recording and analysis of event related potentials," in Handbook of Neurophysiology, Vol. 10 (F. Boller and J. Grafman, eds.), pp. 3-73, Baltimore, Elseviere Science, 1995.

[24] G. G. Celesia and N. S. Peachey, "Visual evoked potentials and electroencephalograms," in Electroencephalography. Basic Principles, Clinical Applications and Related Fields (E. Niedermeyer and F. Lopes da Silva, eds.), pp. 968-993, Baltimore: Williams \& Wilkins, 1999.

[25] J. Polich, "P300 in clinical applications," in Electroencephalography. Basic Principles, Clinical Applications and Related Fields (E. Niedermeyer and F. Lopes da Silva, eds.), pp. 1073-1091, Baltimore: Williams \& Wilkins, 1999.

[26] B. Lutkenhoner and C. Pantev, "Possibilities and limitations of weighted averaging," Biol. Cybern., vol. 52, pp. 409-416, 1985.

[27] C. Davilla and M. Mobin, "Weighted averaging of evoked potentials," IEEE Trans. Biomed. Eng., vol. 39, pp. 338-345, 1992.

[28] C. W. Therrien, Discrete Random Signals and Statistical Signal Processing. New Jersy: Prentice-Hall, 1993.

[29] S. M. Kay, "Spectral Estimation." Advanced Topics in Signal Processing, chapter 2. Prentice Hall, 1993.

[30] A. Oppenheim and R. Schafer, Discrete-Time Signal Processing. Prentice Hall, 1989. 
Signal \& Image Processing : An International Journal (SIPIJ) Vol.2, No.4, December 2011

\section{Authors}

V. Adinarayana Reddy received his graduate degree in Electronics and Telecommunication Engineering from The Institution of Electronics and Telecommunication Engineers, New Delhi in1996 and M. Tech in Electronic Instrumentation and Communication Systems from Sri Venkateswara University, Tirupati in 1999. He joined as faculty in the Department of Electronics and Communication Engineering at KSRM College of Engineering, Cuddapah. Currently he is working as Professor and Head of the Department, Electronics and Communication Engineering at Rajoli Veera Reddy Padmaja Engineering College for Women, Cuddapah, India. His research area of interest includes signal processing and communication systems

Dr Putta Chandra Sekhar Reddy received his B.Tech. degree in Electronics and Communication Engineering from JNTU, Hyderabad, India and M.E. from Bharatiyar University, Coimbatore. He received M.Tech and Ph.D from Jawaharlal Nehru Technological University, Hy derabad, India. He joined as faculty in JNTU, Anantapur. Currently he is working as Professor Co-ordinator in JNTU Hyderabad, India. He is an author of numerous technical papers in the Fields of High speed networking and wireless networks. His research interests include mobile and wireless communications and networks, personal communications service and high speed communications and protocols.

G. Hema Latha received her B.Tech. Degree in Electronics and Communication Engineering from Sri Venkateswara University, Tirupati in 1997, and M.Tech in Instrumentation and Control Systems from Sri Venkateswara Unversity, Tirupati in 2003. She joined as faculty in Electron ics and Communication Engineering at G. Pulla Reddy Engineering College, Kurnool. At present, she is working as Associate Professor in Electronics and Communication Engineering, KSRM College of Engineering, Cuddapah, India. Her research interests include Signal Processing and Communication Systems.

Dr. B. Anuradha received her B.Tech. degree from Gulbarga University, Gulbarga, and M.Tech. and Ph.D. degrees from Sri Venkateswara University, Tirupati. In 1992 She joined as faculty in ECE at Sri Venkateswara University college of Engineering, Tirupati, India. There, she is now working as Associate Professor. She guided many B.Tech and M.Tech. projects. At present four research scholars are working for Ph.D. She had a good number of papers in conferences and published in journals.

Dr. T. Jayachandra Prasad obtained his B.Tech in Electronics and Communication Engg., from JNTU College of Engineering, Anantapur 515002, and Master of Engineering degree in Applied Electronics from Coimbatore Institute of Technology,Coimbatore. He earned his Ph.D. Degree (Complex Signal Processing) in ECE from JNTUCE, Anantapur. He joined as faculty at KSRM College of Engineering, Cuddapah. Later he worked as Head of the department, Electronics \& Communication Engineering, at KSRMCE. Presently he is working as the Principal of RGMCET, Nandyal-518502, India. Dr. Jayachandra Prasad is having more than 22 years of experience and has more than 35 technical publications in National \& International journals and conferences.
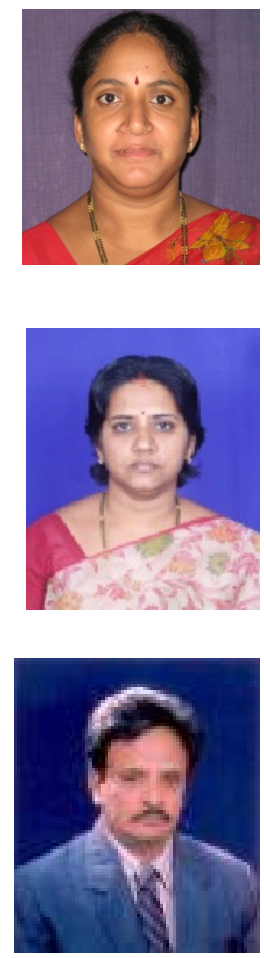